\title{
BATSE Observations of Gamma-Ray Burst Spectra. II. Peak Energy Evolution in Bright, Long Bursts
}

\author{
L. A. Ford, D. L. Band, J. L. Matteson \\ Center for Astrophysics and Space Sciences 0111, University of California at San Diego, \\ La Jolla, CA 92093-0111 \\ M. S. Briggs, G. N. Pendleton, R. D. Preece, W. S. Paciesas \\ University of Alabama at Huntsville, Huntsville, AL 35899 \\ B. J. Teegarden, D. M. Palmer, B. E. Schaefer, T. L. Cline \\ NASA/Goddard Space Flight Center, Code 661, Greenbelt, MD 20771 \\ G. J. Fishman, C. Kouveliotou, C. A. Meegan, R. B. Wilson \\ NASA/Marshall Space Flight Center, ES-62, Huntsville, AL 35812 \\ and \\ J. P. Lestrade \\ Mississippi State University, P.O. Box 5167, \\ Mississippi State, MS 39762
}

\begin{abstract}
We investigate spectral evolution in 37 bright, long gamma-ray bursts observed with the BATSE Spectroscopy Detectors. High resolution spectra are characterized by the energy of the peak of $\nu F_{\nu}$ and the evolution of this quantity is examined relative to the emission intensity. In most cases it is found that this peak energy either rises with or slightly precedes major intensity increases and softens for the remainder of the pulse. Inter-pulse emission is generally harder early in the burst. For bursts with multiple intensity pulses, later spikes tend to be softer than earlier ones indicating that the energy of the peak of $\nu F_{\nu}$ is bounded by an envelope which decays with time. Evidence is found that bursts in which the bulk of the flux comes well after the event which triggers the instrument tend to show less peak energy variability and are not as hard as several bursts in which the emission occurs promptly after the trigger. Several recently proposed burst models are examined in light of these results and no qualitative conflicts with the observations presented here are found.
\end{abstract}

Subject headings: gamma rays: bursts — methods: data analysis 


\section{Introduction}

Gamma-ray burst continuum spectra provide the most direct information about the emission processes involved in these violent events. Unfortunately, the continuum generating process is unknown, making physical interpretation of spectral observations difficult. A striking feature of gamma-ray bursts is the temporal variability of spectra both between and within bursts. Therefore, an empirical study of the dynamics of burst spectra may provide vital clues for resolving the mystery surrounding these puzzling events.

This is the second in a series of reports describing spectral observations of gamma-ray bursts as seen by the Burst and Transient Source Experiment (BATSE) on the Compton Gamma-Ray Observatory (CGRO). The goal of this series is to discover global properties of burst continuum spectra which may shed light on the gamma-ray burst problem.

Gamma-ray bursts remain one of the least understood phenomena in astrophysics despite years of intense study. Their heterogenous nature makes classification difficult and few uniform burst properties are known. Bursts have only been observed above $\gtrsim 2 \mathrm{keV}$ and no emission has been seen in quiescence (Schaefer 1994). In the first paper of this series (Band et al. 1993, hereafter Paper I), spectral properties of time integrated spectra from 54 bright bursts were studied. While spectra are quite diverse, it was shown that a simple and flexible empirical model successfully described all spectra. This model is used in the present study to describe burst spectra and monitor the evolution of the energy at which the energy flux per logarithmic energy band peaks $\left(E_{P}\right.$, energy of the peak of $\left.\nu F_{\nu}\right)$. In future work, similar evolution studies will be performed with other spectral characteristics, such as continuum shape or spectral bandwidth parameters. The data used here are high energy resolution spectra from the Spectroscopy Detectors (SDs) with moderate time resolution $(\geq 0.128 \mathrm{~s})$. Subsequent work will use medium energy resolution data with fine time resolution from the BATSE Large Area Detectors (LADs) to examine weaker bursts and explore techniques using time-tagged counts from the SDs to improve time resolution.

The study of burst spectral evolution has a long history. Golenetskii et al. (1983, hereafter G83) examined two-channel data covering $\sim 40-700 \mathrm{keV}$ with $\sim 0.5 \mathrm{~s}$ time resolution from five bursts observed by the Konus experiment on Venera 13 and 14. Spectra were described by an optically thin thermal bremsstrahlung (OTTB) model, $N_{E}(E) \propto E^{-1} \exp (-E / k T)$ ph-keV ${ }^{-1}-\mathrm{s}^{-1}-\mathrm{cm}^{-2}$. A correlation between luminosity and temperature parameter $T$ was discovered $\left(L \propto T^{\gamma}, \gamma \approx 1.5-1.7\right)$, implying spectral hardness at a given time is related to the intensity of the burst in a simple way. Laros et al. (1985) performed a similar analysis using five bursts observed by the Pioneer Venus Orbiter ( $\sim 0.1-2 \mathrm{MeV}, \sim 0.2 \mathrm{~s}$ time resolution) but found no correlation and speculated that the G83 results were an artifact of the way temperature was inferred. 
Norris et al. (1986, hereafter N86) investigated ten bursts seen by instruments on the Solar Maximum Mission satellite using hardness ratios (the ratio of observed flux in two energy bands). The energy bands used in the hardness ratio were $52-182 \mathrm{keV}$ and 300-350 $\mathrm{keV}$ and the time resolution ranged over 0.128-1s, depending on the data available. They found that individual intensity pulses evolved from hard-to-soft with the hardness peaking before intensity. This implies a more complex relationship between hardness and intensity than proposed by G83.

Recent studies of spectral evolution have tended to follow the lead of G83 or N86, obtaining similar results. Kargatis et al. (1994, hereafter K94) fit OTTB and thermal synchrotron models $\left(N_{E}(E) \propto \exp \left[-\left(E / E_{c}\right)^{1 / 3}\right] \mathrm{ph}-\mathrm{keV}^{-1}-\mathrm{s}^{-1}-\mathrm{cm}^{-2}\right)$ to sixteen bursts from the SIGNE experiment, covering $\sim 50-700 \mathrm{keV}$ with 0.5 second resolution. A luminosity-temperature corelation was found in seven of the bursts $(\gamma \approx 1.4-3.0)$ but two clearly did not show this correlation (the remainder were questionable) indicating that while many bursts may have a luminosity-temperature correlation, the trend is not universal. On the other hand, Band et al. (1992a) analyzed nine bursts observed by the BATSE SDs $(\sim 25-1000 \mathrm{keV}, \geq 0.128 \mathrm{~s})$ using models of the form $N_{E}(E) \propto E^{\alpha} \exp [-(E / k T)]$ ph- $\mathrm{keV}^{-1}-\mathrm{s}^{-1}-\mathrm{cm}^{-2}$, confirming the results of N86 including $T$ leading the intensity when fine time resolution was available. Bhat et al. (1994) used hardness ratios with BATSE LAD data to study single pulse bursts which had a fast rise followed by a gradual decay. The energy bands used for the ratio were $E=25-100 \mathrm{keV}$ and $E>100 \mathrm{keV}$. The time resolution of the sample was $64 \mathrm{~ms}$. Hard-to-soft spectral evolution was found with hardness leading the intensity. The time lag between the peak hardness ratio and peak count rate was found to be directly correlated with the rise time of the counting rate.

Although the analyses exemplified by G83 and N86 give apparently inconsistent results, they can be reconciled by considering the time resolution of the data. The analysis of G83 and K94 had a time resolution of $\sim 0.5 \mathrm{~s}$ while the resolution of N86 was as small as $\sim 0.128 \mathrm{~s}$ and Bhat et al. (1994) had $64 \mathrm{~ms}$ resolution. Therefore, slightly asynchronous behavior could be masked by the poor time resolution in the G83 and K94 samples. This conclusion is supported by the results of Band et al. (1992a), who performed an analysis similar to that of G83 and K94, but found that the spectral hardness (parameterized by $T$ ) led the intensity on short timescales. The need for fine time resolution is emphasized by Kouveliotou et al. (1992), who examined 22 bursts observed with the LADs and performed Fourier transforms on intensity profiles in different energy bands. The hard band was found to lead the soft by $\sim 0.1$ second. In addition, Kouveliotou et al. (1994a) reported significant variations in the hardness ratio at the $2 \mathrm{~ms}$ level in an extremely intense burst observed with BATSE. 
The spectral analyses of G83 and N86 characterize the spectrum in very different ways. Both G83 and K94 use spectral models which are assumed to describe the gamma-ray burst continuum and whose parameters are interpreted as physically meaningful. Most often, the model chosen is one which cuts off rapidly at high energies (e.g., OTTB). It was demonstrated in Paper I that such models do not reproduce spectra observed by the BATSE SDs and conflict with the observation of high energy emission by other experiments (Matz et al. 1985; Schneid et al. 1992; Hanlon et al. 1994). Consequently, these models cannot be physically correct and though the parameters are certainly physically linked to the emission process, one must realize that the parameters do not necessarily have their apparent meaning (e.g., the OTTB cutoff energy may not be temperature). On the other hand, hardness ratios (such as used by N86) do not assume any knowledge of the source physics but do depend on instrumental properties which are accounted for in spectral fitting. While this characterization tracks changes in the shape and slope of a spectrum, the numerical values do not have a quantitative physical meaning and do not lend themselves to additional insights as a characteristic temperature or spectral shape can. Therefore it is helpful to use an empirical model which describes the photon spectrum but does not depend strongly on preconceived notions of spectrum formation in bursts.

In this work, a description of the photon spectrum independent of physical models is achieved by fitting the empirical spectral model from Paper I to BATSE SD data. This model was shown to adequately describe spectra in the BATSE energy range and incorporates many simple physical models as special cases, accommodating uncertainty about the actual continuum emission process. Although the model is phenomenological, physically meaningful parameters can be derived from it. In this work, $E_{P}$ (the energy of the peak of $\nu F_{\nu}$ ) is used to quantify fitted spectra, a parameter which indicates the energy of maximum radiated power. This allows a study of spectral evolution in gamma-ray bursts based on a well-defined physical measure of spectral hardness which does not depend strongly on assumed emission processes.

In this work, the word hardness is used to describe the energy of $E_{P}$. Spectra in which $E_{P}$ is at high energies are called hard and soft spectra have small $E_{P}$. Before discussing the analysis, the SDs are briefly described ( $\$ 2)$. In $\S 3$ the analysis techniques used are presented in detail along with several consistency tests for both the data and methods. The results are presented in $\S 4$ followed by a discussion of their implications for both burst phenomenology and recently proposed theoretical models $(\S 5)$. The entire work is then briefly summarized $(\S 6)$. 


\section{The Instrument}

BATSE is a set of eight detector modules located on the corners of the CGRO spacecraft. Each module contains two detectors: an LAD, optimized for the detection and location of gamma-ray transients, and an SD, optimized for energy resolution. The SDs were designed for spectral studies and are used in this work because of their superior energy resolution to the LADs, which have large area but are thin (20" diameter by $0.5^{\prime \prime}$ thick) $\mathrm{NaI}(\mathrm{Tl})$ crystals.

The gamma-ray detector for an SD is a $5^{\prime \prime}$ diameter by $3^{\prime \prime}$ thick $\mathrm{NaI}(\mathrm{Tl})$ crystal. Because of their thickness, the response to incident gamma-rays is roughly constant over a large range of energies and viewing angles. The SDs independently cover two energy decades in the range $\sim 10 \mathrm{keV}-100 \mathrm{MeV}$ (the exact energy range is determined by a commandable gain setting, with higher gains covering lower energy ranges). The fractional full width at half maximum energy resolution of the SDs at $662 \mathrm{keV}$ is $\sim 7 \%$, with a $\sim E^{-0.4}$ dependence. A $3^{\prime \prime}$ beryllium window in the front of the aluminum case containing the NaI allows the response to extend down to $\sim 5 \mathrm{keV}$ for face-on bursts. In general however, the lower level discriminator is set so that the spectrum is cut-off below $\sim 10 \mathrm{keV}$ in the highest gain setting. An electronic artifact near the low end of spectra (the Spectroscopy detector Low Energy Distortion [SLED], Band et al. 1992b) exists in several channels above the instrument's low energy cutoff which can cause problems for spectral analysis. In this work, only data which are uncontaminated by this artifact are used. The energy deposited in the crystal is analyzed into 2782 linear channels which are rebinned on the spacecraft into 256 quasi-logarithmic channels for transmission to Earth. Despite this compression, the width

of most of the compressed channels is significantly smaller than the detector resolution and little information is lost in this process.

BATSE enters a 4-10 minute long burst mode when the count rate in at least two LADs exceeds the background count rate by a predetermined significance threshold (usually $5.5 \sigma$ ) on one of three timescales: $64 \mathrm{~ms}, 256 \mathrm{~ms}$, and $1024 \mathrm{~ms}$. Several data types are collected in this mode. The type used in this study consists of high energy resolution SD spectra with accumulation time based on a time-to-spill criterion in the LADs (Spectroscopy detector, High Energy Resolution, Burst, called SHERB). When the total number of counts in the LADs exceeds a pre-determined threshold, the current SHERB accumulation ends and the next begins. A total of 192 spectra are recorded from the SDs associated with the four most brightly illuminated LADs. Half of these spectra are from the SD associated with the brightest LAD, one-quarter with the second brightest, and one-eighth each for the other two detectors. The length of the accumulation is a multiple of $64 \mathrm{~ms}$ with a minimum accumulation of $128 \mathrm{~ms}$. Spectra are accumulated until either all spectra are exhausted 
or the burst mode ends. The total time interval covered by each detector is equal: in the time required to accumulate one spectrum in the third and fourth brightest detector, four spectra from the brightest and two spectra from the second brightest detectors are recorded. Additional details about the BATSE detectors can be found in Fishman et al. (1989) and Horack (1991).

\section{Analysis}

It was shown in Paper I that the following empirical model is sufficient to describe burst spectra:

$$
N_{E}(E)\left(\frac{\text { photons }}{{\mathrm{keV}-\mathrm{s}-\mathrm{cm}^{2}}^{2}}\right)= \begin{cases}A\left(\frac{E}{100 \mathrm{keV}}\right)^{\alpha} e^{-E / E_{0}}, & E \leq(\alpha-\beta) E_{0} \\ A^{\prime}\left(\frac{E}{100 \mathrm{keV}}\right)^{\beta}, & E>(\alpha-\beta) E_{0}\end{cases}
$$

where $A, \alpha, \beta$, and $E_{0}$ are fit to observed spectra and $A^{\prime}$ is chosen so that the function is continuously differentiable everywhere. The success of this model can be traced to its flexibility. Included in equation (1) as special cases are a power law, photon exponential, OTTB, and a broken power law with continuous transition. Since the emission process is unknown, equation (1) is a logical choice for determining physically meaningful parameters. The quantity of interest in this study is the energy of the peak of $\nu F_{\nu}\left(E_{P}\right)$ and since $N_{E}(E) E^{2} \propto \nu F_{\nu}, E_{P}=(2+\alpha) E_{0}$ (assuming $\beta<-2$ ).

Burst data were selected from detectors in high gain states, most covering $~ 15-1500 \mathrm{keV}$ with about a third extending to higher energies. This choice was motivated by the results of Paper I which demonstrated that for most bursts the spectral slope changes at energies less than $1 \mathrm{MeV}$ and that the observed signal is greatest around $100 \mathrm{keV}$ (a detector dependent property, not necessarily true for the incident spectrum).

Bursts which had many high quality spectra were required so that $E_{P}$ could be reliably determined and meaningful trends discovered. However, most bursts were not sufficiently long or intense to provide enough spectra to resolve the evolution. To understand the necessary signal strength, a Monte Carlo simulation of a typical spectrum was performed to investigate the reliability of the determination of $E_{P}$ as a function of the signal-to-noise ratio $(\mathrm{S} / \mathrm{N})$ in the $60-200 \mathrm{keV}$ energy band. The results of this simulation are given in Table 1 which shows that a large $\mathrm{S} / \mathrm{N}$ level is required to reduce the variance in $E_{P}$ to reasonable values. 
Even for many strong bursts, most individual SHERB spectra do not have large S/N levels. However, the quality of the signal can be improved by averaging spectra together weighted by livetime. An explicit demonstration of this process for burst 2B910807 is given in Figure 1. In this figure, $E_{P}$ as determined from spectra at the finest time resolution available and from averaged pairs of spectra are plotted together. S/N in the $60-200 \mathrm{keV}$ band ranged from 7.0-17.0 with a median of 11.7 for the fine time resolution data and 9.7-23.1 with median 16.3 for the averaged spectra. It is apparent that not only do the estimated errors in the determination of $E_{P}$ get smaller as spectra are accumulated, but that the fitted value converges to the average of $E_{P}$ determined from the fine resolution spectra.

After selecting candidate bursts, background models were created using a channel-bychannel fit of a time dependent polynomial to data before and after the burst. The order of the polynomial varied from burst to burst; typically the lowest order which gave an acceptable fit was used (never higher than fourth order). After subtracting the background, consecutive spectra were averaged together until $\mathrm{S} / \mathrm{N} \geq 15$ in the $60-200 \mathrm{keV}$ energy band. Although Table 1 shows that this choice will result in a sizable dispersion in $E_{P}$, a larger $\mathrm{S} / \mathrm{N}$ level would have essentially destroyed the time resolution in most of the sample. Therefore, some certainty in $E_{P}$ was sacrificed for time resolution. Exceptions to this $\mathrm{S} / \mathrm{N}$ criterion were made for a few bursts in which a long period of low activity was bounded by interesting pulse structure. In those cases, all data in between the intense periods were averaged despite a low $\mathrm{S} / \mathrm{N}$ value. An exception was also made for burst 2B910813 in which the data did not extend below $100 \mathrm{keV}$. The $\mathrm{S} / \mathrm{N}$ criterion for this burst was $\mathrm{S} / \mathrm{N} \geq 12$ in the 100-200 keV band.

A burst was included in the sample if it survived the process outlined above with at least six spectra. Since most bursts do not have many spectra with large S/N, only $\sim 4 \%$ of all bursts observed by BATSE and 17 of the 54 bursts from Paper I were contained in the sample (this sample includes bursts which occurred after those in Paper I). The empirical model (eqn. [1]) was fitted to data using forward folding deconvolution (Loredo \& Epstein 1989). This method determines the incident photon spectrum by folding an assumed photon spectrum through a model of the detector response and comparing the result to observed data. The best fit was found using a modified version of the Levenberg-Marquardt iterative $\chi^{2}$ minimization algorithm (Bevington 1969, p. 237; Press et al. 1989, p. 521). The modifications were the use of model variances instead of data variances in $\chi^{2}$ (see the appendix for details) and stopping criteria based on $\chi^{2}, \chi^{2}$ per degree of freedom in the fit $\left(\chi_{\nu}^{2}\right)$, and a test for false minima. The detector response model included the direct component along with scatter off the spacecraft (Pendleton et al. 1989) and the Earth's atmosphere (Pendleton et al. 1992). The error in $E_{P}$ was determined through standard 
error propagation using the error in $\alpha$ and $E_{0}$ and the correlation between these two parameters (Bevington 1969, p. 61).

Each spectrum was fit over the entire energy range above the SLED. The data were not rebinned from the telemetered format so that in most cases the average number of counts per bin in the high energy channels was less than a few. In these channels, the Poisson statistical distribution is not well approximated by a normal distribution and the $\chi^{2}$ statistic is therefore not strictly applicable. This is a common difficulty in high energy astrophysics which can be avoided by binning the data until the Gaussian limit is reached. To determine the importance of this problem, a set of Monte Carlo simulations was performed for several types of binning. The results for four different binning modes in Table 1 show that the fits are not improved by rebinning the data even if most high energy bins have only a few counts. There are two reasons for this. First, the data at higher energies are so sparse that when the Gaussian limit is achieved, the bins are so wide that the upper power law in eqn. (1) cannot be accurately determined. Second, the fit is largely determined by the signal below a few hundred $\mathrm{keV}$ where the number of counts per channel is largest $(\operatorname{Max}(\mathrm{C})$ in Table 1$)$. The Gaussian limit can also be achieved if there are a large number of background counts, which can be important at low energies.

These simulations demonstrate that for the SHERB data type used in this sample, any binning is valid. To ensure that this was the case for real data, fits using the telemetered binning and eight broad energy bins were made to spectra from burst 3B930916. The comparison of the values of $E_{P}$ is presented in Figure 2. $E_{P}$ is consistent between the two fits and determined with roughly equal accuracy. Since the choice of data binning is unimportant, the telemetered channels were not rebinned because the analysis software was optimized for this scheme.

One difficulty with using high gain detectors (where the spectrum extends to low energy) was that occasionally the upper spectral index $\beta$ was larger than -2 which implies that $E_{P}$ was beyond the upper end of the fit range, that the spectrum has more gradual curvature than the empirical model could accomodate, or that the high energy signal was insufficient to fix $\beta$. For this work, $\beta$ was constrained to be less than -2 for all fits. In bursts for which this constraint was important (implying a flatter spectrum), it was found that either $\beta$ tended to shift rapidly from the upper constraint to the lower $(\beta=-5)$ or that it remained at the upper constraint $(-2.01)$ consistently. In the former case, the high energy spectrum was probably not well-determined and should be ignored when calculating $E_{P}$. For the latter, $E_{P}$ should reflect changes in the shape (i.e. hardness) of the spectrum since $E_{0}$ will move to higher energies to compensate for the inability of $\beta$ to adequately describe this region. No bursts were observed to show convincing evidence for evolution of 
$\beta$ from above -2 to lower values. Therefore, variations in the fitted value of $E_{P}$ should be consistent with the true variations in spectral hardness if quantitatively inaccurate (with systematically smaller values of $E_{P}$ ). Omitting the upper power law would force $E_{P}$ to higher energies as the model accounts for power at the high end. However, this simpler model may overestimate $E_{P}$, placing it far above the detector's energy range where the model is not as sensitive to changes in $E_{P}$. As a subset of the empirical four parameter model of equation (1), the model without $\beta$ is not a more accurate description of the true photon spectrum. Since evolution of $E_{P}$ is the primary focus of this study, equation (1) with $\beta$ constrained is used to model all spectra since it remains sensitive to hardness variations over a broader energy range than a model which cuts off at high energies.

To test whether changes in the fitted values $E_{P}$ were indeed consistent with real variations in $E_{P}$ for cases where $\beta>-2$, detectors at lower gain were used to determine $E_{P}$ for bursts in which the constraint was important. Unfortunately, in all but one case the high energy signal was too weak to reliably fit $E_{P}$ with any time resolution. Figure 3 shows a comparison of the high and low gain detectors for the exception (2B910503). It is apparent from this figure that not only do the two detectors have similar time histories, but that $E_{P}$ determined by the high gain detector is not far from the value of $E_{P}$ measured by the low gain detector (for which $\beta<-2$ ). Therefore, it is not expected that qualitative statements about the evolution of $E_{P}$ depend strongly on the accuracy with which $\beta$ is determined.

That the low gain detector measures a consistently larger value of $E_{P}$ than the high gain detector in the previous example suggests the calculated value of $E_{P}$ may depend on gain setting even though $E_{P}$ is well defined in all detectors. To ensure that this was not the case, the value of $E_{P}$ from two detectors at different gains viewing burst 2B921207 were compared ( $\beta<-2$ for both detectors). In this case, spectra accumulated by the high gain detector were averaged so that the time intervals covered by both sets of spectra were identical. The comparison in Figure 4 shows that the determination of $E_{P}$ was consistent.

The tests outlined above cover the obvious difficulties involved in the analysis. Although solutions to the $\mathrm{S} / \mathrm{N}$ and $\beta>-2$ problems are not ideal, they do allow the analysis to proceed so that useful information about burst continua can be derived. Also, the inter-detector test indicates that SD observations are consistent with each other and the results should not depend on the variable energy range covered by the SDs.

\section{Results}


Of the 862 gamma-ray bursts detected by BATSE until the end of 1993, only the 37 bursts listed in Table 2 met the criteria outlined above. As expected, equation (1) fit the data well, $\chi_{\nu}^{2} \lesssim 1$ for nearly all spectra. For these bursts, $E_{P}$ was compared to the count rate (see Figure 5). Two bursts which clearly demonstrate the general trends observed in the sample as a whole are 2B921207 and 2B920525. In 2B921207, it is apparent that $E_{P}$ softens over the whole burst except for the increase along with the intensity 8 seconds after the trigger. 2B920525 is a burst in which two strong intensity pulses were resolved. $E_{P}$ softens after intial hardening within both spikes and the second spike is softer than the first.

The plots of Figure 5 were used to characterize types of evolution in $E_{P}$. The classification categories used were motivated by the work of G83 and N86 in which a spectral characteristic (i.e., OTTB temperature and hardness ratio respectively) was tied to changes in burst intensity. These categories describe both the tendency for variations in $E_{P}$ to reflect changes in intensity (G83) and hard-to-soft evolution (N86). The categories are:

1. An increase in $E_{P}$ occurring in proximity to a major intensity increase. $E_{P}$ and intensity need not be morphologically identical and $E_{P}$ can lead or lag the intensity increase by a small amount ( $\lesssim 1 \mathrm{~s})$.

2. General softening of $E_{P}$ in time outside of intensity pulses over the entire burst. Regions which do not have a spike-like intensity profile are compared for this category. Spikes are considered to be short time intervals (less than a few seconds) in which the count rate was much greater than the rate in nearby intervals. For broader pulses, the first few seconds after the maximum count rate was achieved are considered part of the pulse, the remainder was considered inter-pulse emission.

3. Softening of $E_{P}$ within intensity pulse structures. $E_{P}$ should be harder early in the intensity pulse for a burst to fall in this category. It can be measured only if the pulse is temporally resolved.

4. Later intensity pulses have a softer peak in $E_{P}$ than earlier pulses. This can only be measured if the burst has multiple intensity spikes.

Bursts were judged as showing the property in question, showing an opposite trend, or showing neither trend $(+,-$, and 0 respectively in Table 2). To demonstrate how a burst was categorized, consider 2B921207. This burst satisfies the first category because $E_{P}$ hardens as the intensity increases at 0.5 and 8 seconds after the trigger. The interpulse region lies between 2.5 and 8 seconds, where $E_{P}$ clearly softens. Both intensity spikes 
(0.5-2.5 seconds and 8-10 seconds after trigger) are temporally resolved and $E_{P}$ softens in each. Finally, the peak $E_{P}$ is softer in the later spike than in the first.

The rating system used here is admittedly simplistic, but is preferable to schemes which allow for finer distinctions within a class (e.g., rating bursts on a scale of 1-10, either discretely or continuously) for two reasons. First, the sample size is small so that the subdivision of bursts into several discrete subcategories reduces the significance of the classification. Second, the mediocre time resolution for some time intervals and sizable errors for some $E_{P}$ create problems for a continuous classification scheme. Therefore, although the criteria outlined above are subjective, they are the least problematic given the state of the observations.

Individual bursts are detailed in Table 2 and characteristics for the sample as a whole are given in Table 3. Included in Table 2 are the median values of $E_{P}$ and the range in $E_{P}\left[\Delta E_{P} \equiv \max \left(E_{P}\right)-\min \left(E_{P}\right)\right]$ for each burst. Not all bursts could be classified because of large errors in $E_{P}$, unresolved structure, lack of structure, poor time resolution, etc. Bursts in which $\beta$ was fit consistently at the upper constraint are noted in Table 2 as are bursts which were simultaneously observed in the 1-30 MeV range with the COMPTEL instrument on $C G R O$ (Hanlon et al. 1994). In all four cases, the fitted $\beta$ was consistent with power law fits to the COMPTEL data in that for bursts in which COMPTEL reported a power law of index $>-2, \beta$ was consistently fit to the upper constraint. It can be seen from Figure 5 that there are several instances in which $E_{P}$ was poorly fit resulting in a large error $\left(\sigma_{E_{P}}\right)$ for this quantity. Spectra for which $\sigma_{E_{P}} \gtrsim 0.5 E_{P}$ were ignored when characterizing bursts and calculating $\Delta E_{P}$.

Table 2 clearly demonstrates that increases in $E_{P}$ are associated with rises in intensity and examination of Figure 5 shows that in many cases, the peak in $E_{P}$ leads the intensity peak. A quantitative analysis of $E_{P}$ leading intensity was not performed since Kouveliotou et al. (1992) found the typical lead time is shorter then the resolution of this sample. In addition, the time profile is extremely complex on short timescales so that a detailed study would best be performed using data with better time resolution. This data can be obtained from the LADs and the analysis is currently being performed (Kouveliotou et al. 1994b). Table 3 shows that many bursts undergo hard-to-soft evolution both within and outside of intensity spikes as well as between successive pulses. Softening of successive spikes is explicitly demonstrated in Figure 6, where the peak in $E_{P}$ for early intensity spikes is compared to the same quantity in later spikes in multi-spike bursts. It can be seen that late pulses are more likely to be softer than those which occurred previously, suggesting that the maximum possible value for $E_{P}$ is bounded by an envelope decaying in time. The trends noted above suggest that spectral hardness leads the intensity modulated by a decay 
envelope.

It should be mentioned that there are bursts in the sample which do not share the traits outlined above. This shows that while typical burst characteristics may have been identified, they are not universal on the timescale at which this sample was studied. However, it is not unreasonable to expect that $E_{P}$ leading intensity and the softening trends noted above will be found in most bursts when observed at finer time resolution.

The general softening trends in $E_{P}$ imply that the time at which emission occurs relative to the beginning of the burst is an important factor in spectral evolution. This raises an interesting question: assuming that the maximum values of $E_{P}$ are bounded by an envelope decaying in time, then if a burst has a precursor several seconds before most of the emission occurs, does this envelope begin to decay when the precursor happens or is the precursor irrelevant with respect to spectral evolution? To examine this question, the range in $E_{P}$ and the time at which significant emission occurred relative to the BATSE trigger were compared (Figure 7). Significant emission was defined as emission capable of producing spectra which met the $\mathrm{S} / \mathrm{N}$ criterion outlined in $\S 3$. In Figure 7 the horizontal bar indicates the period of significant emission. It begins at $t_{d}$, the time delay between the instrument trigger and the beginning of significant emission, and has length equal to the duration of significant emission $(\Delta T)$. A large value of $t_{d}$ results when a long period of time passes between the trigger (a burst precursor) and the brightest emission. The data in Figure 7 occupy a triangular region indicating the sample was devoid of bursts with hard, highly evolving spectra which began emitting significantly well after the BATSE trigger: the few bursts with large $t_{d}$ have small values of $E_{P}$ and $\Delta E_{P}$. To determine the importance of these bursts, the sample was divided into four groups based on the values of $t_{d}$ and $\Delta E_{P}$. Since there are no clear groupings in Figure 7, the divisions were $t_{d}=0$ (significant emission at trigger) and $\Delta E_{P}=400 \mathrm{keV}$, which each divide the sample in half, producing an unbiased division. As shown by Table 4 , bursts with $t_{d} \neq 0$ do tend to evolve less, but the significance of this result is small $(\sim 1 \sigma)$, implying that the apparent $t_{d}-\Delta E_{P}$ association may be a statistical aberration which will vanish when more bursts with large $t_{d}$ are observed.

For several bursts, the nonzero time delay was short compared to the duration of significant emission. This suggests that the measure of time delay should be the ratio of $t_{d}$ and $\Delta t$. This definition is preferable to the absolute measure since it incorporates the timescale of the event from the trigger to the end of significant emission, allowing comparisons based on the time the burst began its most active stages and the total length of the burst. Bursts were divided into two groups based on the value of $t_{d} / \Delta t$. Table 5 shows the number of bursts in each group for several values of this ratio as well as the median and extreme values of $\Delta E_{P}$ for each group. The table shows that late emitters tend to 
show less $E_{P}$ variability than bursts which emit promptly after the trigger. Although this is very suggestive, there is sizable overlap between the groups. Considering the small size of the sample, this result is not too significant and therefore the possibility of a dependence of $\Delta E_{P}$ on $t_{d}$ should be treated with some caution.

\section{Discussion}

In this work it was shown that for most gamma-ray bursts, the energy of the peak of $\nu F_{\nu}$ is associated with and sometimes leads the intensity of the burst modulated by an envelope decaying in time. Indications that bursts whose main emission occurs at relatively long times after the trigger are generally soft and tend to evolve less in $E_{P}$ were also found. In this section the phenomenological implications of these results are discussed. Some recently proposed burst models are also examined in light of the observations reported here and no qualitative conflicts between the models considered and observations are found.

\subsection{Phenomenological Implications}

While it has been known for some time that burst spectra tend to evolve from hard to soft (N86), the observations presented here show that the time of emission relative to the beginning of the burst may also determine how the spectral hardness evolves. For optically thin burst models (in which particles radiate on timescales much shorter than the resolution of SHERB spectra), these results imply that as the burst transpires, the emission region 'remembers' previous emission suggesting that either emission comes from only one region or that different emission regions must be physically connected. The softening of successive intensity pulses could be caused by significant change in the emitting region during intense episodes (such as an expansion or an increase in the number density of particles). The softness of delayed emission implies that the emission region can evolve without observable radiation. For optically thick models, a burst could be the result of a single energy input since the radiation does not escape the region on short timescales. If the region cools by adiabatic expansion and the surface of last scattering does not propagate inward to higher temperature regions too quickly, then observable radiation would be expected to evolve from hard-to-soft. The softness of delayed emission could also be explained in this way if the region was not able to emit significantly during the initial stages of expansion. 
The hard-to-soft spectral evolution within pulse structures indicates that intensity spikes are not symmetric in time. Nemiroff et al. (1994b) reached a similar conclusion by examining the time structure of intensity profiles. These observations clearly show that time symmetric models of spectra formation as well as overall intensity profiles cannot be correct. An example of the kind of model eliminated by these observations is symmetric beam models in which the beam sweeps by much faster than the timescale over which the beam changes.

Another implication of this work relates to burst duration classes. The distribution of gamma-ray burst durations observed by BATSE is bimodal and short bursts (duration $\lesssim 2 \mathrm{~s}$ ) tend to have larger hardness ratios than longer ones (Kouveliotou et al. 1993; Lamb et al. 1994) where the hardness ratios were averaged over the whole burst. Based on the large values of $\Delta E_{P}$ reported here and the fact that the hardest emission tends to come early in a burst, short bursts are expected to be harder than long ones since they do not have softer tails, not necessarily because of an inherent difference in the radiation mechanism. An example of the effect of time averaging is burst 2B920525. If spectra from 4.16-6.72 s (the first spike) are used $E_{P}=650 \pm 70 \mathrm{keV}$ but falls to $421 \pm 25 \mathrm{keV}$ if the entire burst is integrated into one spectrum. As Norris et al. (1994) also suggested, if hardnesses are measured over the same absolute time interval (e.g., compare short bursts to the first few seconds of long bursts), the hardness distribution may be the same for both classes.

In Paper I it was shown that $E_{P}$ varied considerably from burst to burst indicating that there is no universal characteristic energy of burst emission. One conclusion which was drawn from this was that pair processes could be directly observed only if burst sources had a broad range of redshifts. Such processes would produce spectra with an annihilation feature at $511 \mathrm{keV}$ and would cut off above this energy because of pair opacity. The observed energy of these features depends on the redshift of the source region. The argument against observable pair processes can be extended by noting the large changes in $\Delta E_{P}$ observed within many bursts in this sample (Table 3). Unless the emission process involves large and rapid changes in redshift, pair processes cannot be directly observed in burst spectra.

The data in Figure 7 include several bursts in which the emission was prompt but had small $\Delta E_{P}$. An interesting possibility is that these prompt emitting, relatively non-evolving bursts were preceded by a precursor too faint to trigger BATSE. In this case, bursts which occupy the lower left-hand corner of the figure are late emitters for which BATSE did not trigger on the initial event. A less exciting possibility is that bursts which emit late but evolve considerably exist and there is no relation between emission time and $\Delta E_{P}$. Although this type of behavior is not seen, it cannot be ruled out because of the small 
sample size and rarity of late emitters (about one strong burst per year with $t_{d}>60 \mathrm{~s}$ ). Therefore, this may remain an open question even if BATSE detects many bursts over the next few years.

\subsection{Implications for Burst Models}

A plethora of gamma-ray burst scenarios have been proposed (Nemiroff 1994a). However, most attempt to explain only general spectral characteristics and energetics with little or no mention of how spectra are expected to evolve. Therefore, several models are examined to find those which can accommodate the observations presented here. Because BATSE observations of burst isotropy and inhomogeneity have cast serious doubt on older models, only those proposed after 1991 (the year BATSE was launched) are considered. Discussion is further restricted to models which purport to explain all or most gamma-ray bursts. Although arguments have been made for two population distributions (Lingenfelter \& Higdon 1992; Smith \& Lamb 1993), the inability to separate bursts into two uncontroversial classes makes such discussion premature. Jet models are also avoided (c.f. Brainerd 1992; Dermer \& Schlickeiser 1994) since the phenomenon is poorly understood and the models are incomplete. 'Exotic' sources such as cosmic strings (Paczyński 1988), strange stars (Haensel et al. 1991), or primordial black holes (Cline \& Hong 1992) are also omitted since the existence of such sources is highly speculative.

The distribution of bursts seen by BATSE has forced bursts out to extended halo or cosmological distances if they arise from a single source population. Since source regions are inferred to be small from the fast rise times of bursts, the energy density at the source must be enormous, particularly if the burst radiates isotropically. Therefore, many models incorporate optically thick relativistic electron-positron fireballs. As originally proposed (Goodman 1986; Paczyński 1986), fireballs cannot explain the observed nonthermal emission and will not be relativistic if the fireball is contaminated by baryons (Cavallo \& Rees 1978; Paczyński 1990).

Many different scenarios have been proposed recently (c.f. Narayan, Paczyński, \& Piran 1992; Usov 1992; Woosley 1992) which can essentially be reduced to a fireball which must either form in a region free of baryons or break through a cloud of baryons to be observed. In these cases, the observed nonthermal spectra are produced either by several thermal blackbodies at different temperatures (corresponding to different fireballs) or through interactions with a strong or turbulent magnetic field. If a number of fireballs occur, then hard-to-soft spectral evolution could be explained by the creation of low 
temperature fireballs or rapid cooling as the burst transpires. For magnetic interaction models, the general softening of $E_{P}$ may reflect a gradual decline in available energy as the field evolves into a stable configuration.

Mészáros \& Rees (1992) have proposed a model involving a baryon contaminated fireball from any source, galactic or cosmological. The baryons in the fireball interact with the interstellar medium (ISM) to form a relativistic shock where gamma-rays are radiated. Hard-to-soft evolution could be explained either by the most energetic baryons reaching the shock first or the gradual decline of average energy as more particles encounter the shock. The softness of late emission could be a consequence of the baryon front losing energy to the ISM before the shock is fully formed or could arise from the shock losing energy as it propagates through the ISM.

In a different type of model suggested by Melia \& Fatuzzo (1992), a gamma-ray burst is generated by sheared Alfvén waves from a radio pulsar. The waves are created by crustal disturbances at the polar cap which flood the magnetosphere with charged particles. These charges upscatter ambient radio photons to gamma-ray energies which are beamed outward. Hard-to-soft evolution may result from the most energetic charges scattering first and successive spikes soften if subsequent disturbances are less energetic than the original disturbance. Since the emission is beamed, the hard emission might occur when the beam was not pointed in our direction. Delayed emission might be soft because the early hard emission was not beamed in our direction.

\section{Summary}

In this work, the evolution of the energy of the maximum flux per logarithmic energy band $\left(E_{P}\right)$ in long, bright gamma-ray bursts was studied using high energy resolution spectra from the BATSE SDs. It was found that $E_{P}$ is coupled with the intensity of a burst (either leading or accompanying intensity increases) but is apparently modulated by an envelope decaying in time. These results are consistent with previous studies by N86 and Bhat et al. (1994). Indications were also found that bursts in which significant emission is substantially delayed relative to the instrument trigger tend to be soft and evolve less in $E_{P}$ although the significance of this result is low. Several burst models were examined in light of these results and it was found that none of those discussed could be ruled out on the basis of these results alone. However, as the models mature to the point of more precise spectral predictions, the observations presented here may prove to be a useful constraint or suggest directions for further effort. 
We thank D. Gruber, J. Higdon, R. Lingenfelter, and R. Rothschild for helpful advice and useful information. D. Marsden and D. Potter provided additional assistance. BATSE work at UCSD is supported by NASA contract NAS8-36081.

\section{A. Model Weights for $\chi^{2}$}

The Levenberg-Marquardt algorithm (Bevington 1969, p. 237; Press et al. 1989, p. 521 ) is a prescription to efficiently minimize goodness-of-fit statistics. The most common statistic used is

$$
\chi^{2}=\sum_{i=1}^{N}\left[\frac{y_{i}-y\left(x_{i} ; \mathbf{a}\right)}{\sigma_{i}}\right]^{2},
$$

where: $x$ is the independent variable (here energy); $y$ is an observed variable (count rate in this case); $y\left(x_{i} ; \mathbf{a}\right)$ is the value of the model at $x_{i}$ for parameters $\mathbf{a} ; N$ is the number of measurements (here, channels in a spectrum); a represents the parameters to be fit; and $\sigma_{i}$ are the errors associated with $y_{i} \cdot \chi^{2}$ is minimized using an iterative gradient procedure for which the first and second partial derivatives of $\chi^{2}$ with respect to a are needed. In its usual implementation, the algorithm assumes that the $\sigma_{i}$ are taken directly from the data and are constant.

The problem with using data variances $\left(\sigma_{i}\right)$ in equation $(\mathrm{A} 1)$ is that $\sigma_{i}$ weights downward fluctuations in the data too strongly, a result of the Poisson nature of count data in which $\sigma_{i}^{2} \propto n_{i}$, the number of observed counts. In this case, a small number of counts create a smaller than average $\sigma_{i}$ which causes the associated data points to be more important in the calculation of $\chi^{2}$. Wheaton et al. (1994, hereafter W94) examined this problem in detail using a Monte Carlo simulation of 24,000 one-parameter data sets and found that the mean of fitted values deviated from the true value by $-170 \sigma$ (note the downward shift). The solution to this problem is to use $\sigma_{i}$ generated from the model under consideration rather than the data, replacing $\sigma_{i}^{2} \propto n_{i}$ with $\sigma_{i}^{2}\left(x_{i} ; \mathbf{a}\right) \propto n_{i}\left(x_{i} ; \mathbf{a}\right)$ (model variance). When W94 performed the simulation with this new scheme, the bias was $-0.34 \sigma$.

Although W94 demonstrated the importance of using weights derived from the model, they do not mention a necessary change in the standard Levenberg-Marquardt prescription to accommodate the new weighting. The algorithm assumes that $\sigma_{i}$ is taken from the data so that changes in the $\chi^{2}$ surface are contained in only the $y\left(x_{i} ; \mathbf{a}\right)$ term in equation (A1). However, by making $\sigma_{i}$ model dependent without changing the partial derivatives, assumed changes in the $\chi^{2}$ surface caused by variation in a will be incorrect, resulting in slightly

off-course iterations and an incorrect minimum since $\partial \chi^{2} / \partial \mathbf{a}=0$ can be at the wrong value 
of a. It was found that when this effect is not accounted for, the true minimum of the $\chi^{2}$ surface is not reached and the values of the fitted parameters differ from the true best fit by roughly the quoted error in the fitted parameters though it is not biased toward higher or lower values making it difficult to detect in large collections of fits.

Alterations in the second derivative terms only affect the route used to find the minimum of $\chi^{2}$ and therefore need not be corrected for the new weighting scheme Press et al. 1989, p. 523). On the other hand, the first partial derivatives need to reflect any changes which might occur in the $\chi^{2}$ surface and must be corrected. The corrected version is

$$
\frac{\partial \chi^{2}}{\partial a_{j}}=-2 \sum_{i=1}^{N}\left[\frac{y_{i}-y_{i}\left(x_{i} ; \mathbf{a}\right)}{\sigma_{i}^{2}\left(x_{i} ; \mathbf{a}\right)}\right]\left(\frac{\partial y_{i}\left(x_{i} ; \mathbf{a}\right)}{\partial a_{j}}-\frac{\left[y_{i}-y_{i}\left(x_{i} ; \mathbf{a}\right)\right]}{\sigma_{i}\left(x_{i} ; \mathbf{a}\right)} \frac{\partial \sigma_{i}\left(x_{i} ; \mathbf{a}\right)}{\partial a_{j}}\right) .
$$

The first partial derivative term in this equation is equal to the partial derivative specified by the Levenberg-Marquardt algorithm with model variances replacing data variances but the second term is new. For this work, equation (A2) can be expressed in a simpler form since background subtracted count rates are used $\left(y_{i}=n_{i} / f_{i}-b_{i}\right.$ where $f_{i}$ is a term which converts counts to a count rate and $b_{i}$ is the background counting rate). In this case, $\sigma_{i}^{2}\left(x_{i} ; \mathbf{a}\right)$ becomes

$$
\sigma_{i}^{2}\left(x_{i} ; \mathbf{a}\right)=\frac{y_{i}\left(x_{i} ; \mathbf{a}\right)}{f_{i}}+\sigma_{b_{i}}^{2},
$$

where $\sigma_{b_{i}}^{2}$ is the error in the determination of the background rate in channel $i$. Differentiating (A3) with respect to a and substituting into equation (A2), it is found that

$$
\frac{\partial \chi^{2}}{\partial a_{j}}=-\sum_{i=1}^{N}\left[y_{i}-y_{i}\left(x_{i} ; \mathbf{a}\right)\right] \frac{\sigma_{i}^{2}+\sigma_{i}^{2}\left(x_{i} ; \mathbf{a}\right)}{\sigma_{i}^{4}\left(x_{i} ; \mathbf{a}\right)} \frac{\partial y_{i}\left(x_{i} ; \mathbf{a}\right)}{\partial a_{j}} .
$$

This expression is equal to the uncorrected expression when $\sigma_{i}\left(x_{i} ; \mathbf{a}\right)$ is replaced by $\sigma_{i}$ which allows the standard algorithms (e.g. Bevington 1969, p. 237; Press et al. 1989, p. 521) to be modified easily. When this expression is substituted into the algorithm, the true minimum of the $\chi^{2}$ surface with model variances is found. 
Table 1. Monte Carlo Simulations

\begin{tabular}{ccccccc}
\hline \hline Binning $^{1}$ & $t(\mathrm{~s})^{2}$ & $\operatorname{Med}(\mathrm{C})^{3}$ & $\operatorname{Max}(\mathrm{C})^{4}$ & $\operatorname{Med}\left(E_{P}\right)^{5}$ & $\operatorname{Var}\left(E_{P}\right)^{6}$ & $\mathrm{~S} \mathrm{~N}^{7}$ \\
\hline \multirow{2}{*}{ nobin } & 0.1 & 0.23 & 4.66 & 259 & 286 & 10.0 \\
& 0.25 & 0.99 & 7.78 & 285 & 77 & 15.6 \\
& 0.5 & 2.11 & 16.48 & 265 & 50 & 21.6 \\
& 1.0 & 4.41 & 30.31 & 251 & 36 & 29.6 \\
& 2.0 & 9.70 & 43.72 & 250 & 21 & 39.5 \\
\hline half & 0.1 & 1.18 & 9.52 & 271 & 176 & 10.0 \\
& 0.25 & 2.78 & 26.85 & 259 & 85 & 15.6 \\
& 0.5 & 4.66 & 45.71 & 256 & 56 & 21.6 \\
& 1.0 & 9.05 & 86.85 & 252 & 39 & 29.6 \\
& 2.0 & 21.50 & 219.33 & 251 & 22 & 39.5 \\
\hline full & 0.1 & 2.46 & 15.47 & 252 & 153 & 10.0 \\
& 0.25 & 4.95 & 36.03 & 244 & 68 & 15.6 \\
& 0.5 & 8.55 & 72.05 & 253 & 50 & 21.6 \\
& 1.0 & 19.30 & 141.77 & 251 & 35 & 29.6 \\
& 2.0 & 45.47 & 321.04 & 250 & 21 & 39.5 \\
\hline eight & 0.1 & 3.29 & 10.52 & 220 & 86 & 10.0 \\
& 0.25 & 8.54 & 24.35 & 240 & 63 & 15.6 \\
& 0.5 & 17.67 & 46.76 & 247 & 46 & 21.6 \\
& 1.0 & 34.85 & 102.28 & 250 & 40 & 29.6 \\
& 2.0 & 73.27 & 195.23 & 248 & 25 & 39.5 \\
& & & & & & \\
\hline
\end{tabular}

${ }^{1}$ The binning scheme used for spectra:

nobin - Data in native format.

half - Binned to one half of the detector energy resolution.

full - Binned to the detector energy resolution.

eight - Eight broad energy bins.

${ }^{2}$ The livetime used to create spectra.

${ }^{3}$ Median number of counts per channel in the binned spectrum.

${ }^{4}$ Maximum number of counts per channel in the binned spectrum.

${ }^{5}$ Median value of $E_{P}(\mathrm{keV})$.

${ }^{6}$ Variance of $E_{P}$ about the median $(\mathrm{keV})$.

${ }^{7}$ Median S/N in the 60-200 keV energy band.

Note. - 200 spectra were created using the model spectrum of eqn. (1) with $A=0.1 \mathrm{ph}-\mathrm{keV}^{-1}-\mathrm{s}^{-1}-\mathrm{cm}^{-2}, \alpha=-1, \beta=-3, E_{0}=250 \mathrm{keV}\left(E_{P}=250 \mathrm{keV}\right)$. Nonintegral counts result from subtraction of fractional background counts. 
Table 2. Classification of the Sample

\begin{tabular}{|c|c|c|c|c|c|c|}
\hline \multirow[b]{2}{*}{ Burst } & \multicolumn{4}{|c|}{ Characteristic } & \multirow{2}{*}{$\begin{array}{c}\operatorname{Med}\left(E_{P}\right) \\
(\mathrm{keV})\end{array}$} & \multirow{2}{*}{$\begin{array}{r}\Delta E_{P} \\
(\mathrm{keV})\end{array}$} \\
\hline & 1 & 2 & 3 & 4 & & \\
\hline 2B910503*† & + & + & + & + & 540 & 1350 \\
\hline $2 \mathrm{~B} 910601^{\dagger}$ & + & 0 & $\ldots$ & $\ldots$ & 700 & 950 \\
\hline 2B910807 & + & 0 & + & 0 & 170 & 175 \\
\hline 2B910814 & $\ldots$ & $\ldots$ & $\ldots$ & $\ldots$ & 490 & 900 \\
\hline $2 \mathrm{~B} 910814 \mathrm{C}^{\dagger}$ & $\cdots$ & + & $\cdots$ & $\ldots$ & 1400 & 1300 \\
\hline 2B911031* & $\ldots$ & + & $\ldots$ & $\cdots$ & 400 & 850 \\
\hline 2B911106 & $\cdots$ & 0 & $\cdots$ & $\cdots$ & 165 & 120 \\
\hline $2 \mathrm{~B} 911118^{\dagger}$ & + & + & + & + & 180 & 440 \\
\hline 2B911126* & + & + & + & 0 & 250 & 900 \\
\hline 2B911127 & $\cdots$ & $\cdots$ & $\cdots$ & $\cdots$ & 190 & 250 \\
\hline 2B911202* & 0 & + & 0 & $\ldots$ & 350 & 1300 \\
\hline 2B911209* & + & + & + & + & 270 & 400 \\
\hline 2B920218* & $\cdots$ & + & $\cdots$ & $\cdots$ & 220 & 150 \\
\hline 2B920311 & + & + & + & + & 440 & 1150 \\
\hline 2B920406* & + & 0 & + & 0 & 240 & 300 \\
\hline 2B920513* & + & $\cdots$ & + & $\cdots$ & 220 & 200 \\
\hline 2B920517* & + & $\cdots$ & + & $\cdots$ & 230 & 150 \\
\hline 2B920525* & + & + & + & + & 380 & 2075 \\
\hline 2B920617 & $\cdots$ & + & $\cdots$ & $\cdots$ & 145 & 175 \\
\hline 2B920622 & + & 0 & + & $\cdots$ & 440 & 1550 \\
\hline 2B920627* & $\cdots$ & $\ldots$ & $\ldots$ & $\ldots$ & 210 & 325 \\
\hline 2B920711 & + & 0 & $\ldots$ & 0 & 510 & 650 \\
\hline 2B920720 & + & - & - & - & 270 & 525 \\
\hline 2B920902* & $\cdots$ & + & + & $\cdots$ & 550 & 1500 \\
\hline 2B921009 & + & 0 & $\cdots$ & $\cdots$ & 300 & 225 \\
\hline 2B921123 & + & + & $\ldots$ & $\cdots$ & 250 & 350 \\
\hline 2B921207 & + & + & + & + & 140 & 650 \\
\hline 2B921209 & + & + & + & + & 180 & 375 \\
\hline 2B930120 & $\cdots$ & $\cdots$ & $\cdots$ & $\cdots$ & 125 & 80 \\
\hline 3B930405 & $\cdots$ & - & $\cdots$ & $\cdots$ & 280 & 300 \\
\hline 3B930425 & $\cdots$ & $\cdots$ & $\cdots$ & $\cdots$ & 250 & 225 \\
\hline 3B930506 & + & + & 0 & $\cdots$ & 1030 & 1750 \\
\hline 3B930916* & 0 & 0 & 0 & 0 & 390 & 350 \\
\hline 3В930922 & + & + & + & 0 & 110 & 130 \\
\hline
\end{tabular}


Table 2-Continued

\begin{tabular}{ccccccc}
\hline \hline Burst & \multicolumn{3}{c}{ Characteristic } & & $\operatorname{Med}\left(E_{P}\right)$ & $\Delta E_{P}$ \\
& 1 & 2 & 3 & 4 & $(\mathrm{keV})$ & $(\mathrm{keV})$ \\
\hline \multirow{2}{*}{ 3B931103 } & + & + & + & + & 380 & 1300 \\
3B931126 & + & + & + & + & 240 & 300 \\
3B931204 & + & + & + & 0 & 380 & 775 \\
& & & & & & \\
\hline
\end{tabular}

${ }^{*} \beta=-2.01$ throughout the burst.

†Observed by COMPTEL ( Hanlon et al. 1994).

Burst Characteristics:

$1-E_{P}$-intensity association.

2 - Overall softening within a burst.

3 - Softening within pulse structures.

4 - Later spikes softer than earlier spikes.

The symbols denote:

+ - Trait observed.

- - Opposite behavior observed.

0 - Neither trait nor opposite observed.

... - Could not be determined. 
Table 3. Summary of Classification 37 Bursts

\begin{tabular}{ccl}
\hline \hline \# Observed & \# Possible & \multicolumn{1}{c}{ Property } \\
\hline 23 & 25 & $E_{P^{-}}$intensity association \\
20 & 30 & $E_{P}$ softens over whole burst \\
2 & 30 & $E_{P}$ hardens over whole burst \\
18 & 22 & $E_{P}$ softens within intensity spikes \\
1 & 22 & $E_{P}$ hardens within intensity spikes \\
9 & 17 & Later spikes softer than earlier ones \\
1 & 17 & Later spikes harder than earlier ones \\
\hline
\end{tabular}


Table 4. Unbiased Burst Separation

\begin{tabular}{ccc}
\hline \hline & $\Delta E_{P}<400 \mathrm{keV}$ & $\Delta E_{P} \geq 400$ \\
\hline$t_{d} \neq 0$ & 10 & 7 \\
$t_{d}=0$ & 8 & 12 \\
& & \\
\hline
\end{tabular}

Note. - This table shows little evidence that bursts in which emission is delayed evolve differently than those which emit promptly. However, although the criteria used to divide the sample is unbiased, it may not be appropriate since most bursts emit very soon after the trigger. 
Table 5. Bursts Divided According to Emission Time

\begin{tabular}{rcccc}
\hline \hline$\frac{t_{d}}{\Delta T}$ & Number & $\begin{array}{c}\operatorname{Min}\left(\Delta E_{P}\right) \\
(\mathrm{keV})\end{array}$ & $\begin{array}{c}\operatorname{Med}\left(\Delta E_{P}\right) \\
(\mathrm{keV})\end{array}$ & $\begin{array}{c}\operatorname{Max}\left(\Delta E_{P}\right) \\
(\mathrm{keV})\end{array}$ \\
\hline$>0.25$ & 10 & 80 & 300 & 950 \\
$\leq 0.25$ & 27 & 120 & 525 & 2075 \\
\hline$>0.5$ & 8 & 80 & 300 & 950 \\
$\leq 0.5$ & 29 & 120 & 525 & 2075 \\
\hline$>0.9$ & 7 & 80 & 300 & 950 \\
$\leq 0.9$ & 30 & 120 & 525 & 2075 \\
\hline$>1$ & 5 & 80 & 300 & 950 \\
$\leq 1$ & 32 & 120 & 440 & 2075 \\
& & & & \\
\hline
\end{tabular}

Note. - This table summarizes the differences in evolution of $E_{P}$ for bursts grouped according to the time at which they emit. 


\section{REFERENCES}

Band, D. L., et al. 1992, in Gamma Ray Bursts, eds. W. S. Paciesas and G. J. Fishman (New York: AIP), p. 169

Band, D. L., et al. 1992, Exp. Astron., 2, 307

Band, D. L., et al. 1993, ApJ, 413, 281

Bevington, P. R. 1969, Data Reduction and Error Analysis for the Physical Sciences, (New York: McGraw-Hill)

Bhat, N. P., Fishman, G. J., Meegan, C. A., Wilson, R. B., Kouveliotou, C., Paciesas, W. S., Pendleton, G. N., \& Schaefer, B. E. 1994, ApJ, 426, 604

Brainerd, J. J. 1992, ApJ, 394, L33

Cavallo, G. \& Rees, M. J. 1978, MNRAS, 183, 359

Cline, D. B., \& Hong, W. 1992, ApJ, 401, L57

Dermer, C. D., \& Schlickeiser, R. 1994, in Proc. of the Huntsville Gamma-Ray Burst Workshop, eds. G. J. Fishman, K. C. Hurley, \& J. J. Brainerd (New York: AIP), in press

Fishman, G. J., et al., 1989, in Proc. Gamma Ray Observatory Science Workshop (Washington: NASA), 2-39

Fishman, G. J., et al., 1994, ApJS, in press

Golenetskii, S. V., Mazets, E. P., Aptekar, R. L., \& Ilyinskii, V. N. 1983, Nature, 306, 451

Goodman, J. 1986, ApJ, 308, L47

Haensel, P., Paczyński, B., \& Amsterdamski, P. 1991, ApJ, 375, 209

Hanlon, L. O., et al. 1994, A\&A, in press

Horack, J. M. 1991, Development of the Burst and Transient Source Experiment, NASA reference publication 1268

Kargatis, V. E., Liang, E. P., Hurley, K. C., Barat, C., Eveno, E., \& Niel, M., ApJ, 422, 260 
Kouveliotou, C., Norris, J. P., Fishman, G. J., Meegan, C. A., Wilson, R. B., \& Paciesas, W. S. 1992, in Gamma Ray Bursts, eds. W. S. Paciesas and G. J. Fishman (New York: AIP), p. 299

Kouveliotou, C., Meegan, C. A., Fishman, G. J., Bhat, N. P., Briggs, M. S., Koshut, T. M., Paciesas, W. S., \& Pendleton, G. N. 1993, ApJ, 413, L101

Kouveliotou, C., et al. 1994, ApJ, 422, L59

Kouveliotou, C. et al. 1994, in preparation

Lamb, D. Q., Graziani, C., \& Smith, I. A. 1994, ApJ, in press

Laros, J. G., et al. 1985, ApJ, 290, 728

Lingenfelter, R. E. \& Higdon, J. C. 1992, Nature, 356, 132

Loredo, T. J. \& Epstein, R. I. 1989, ApJ, 336, 896

Matz, S. M., Forrest, D. J., Vestrand, W. T., Chupp, E. L., Share, G. H., \& Rieger, E. 1985, ApJ, 288, L37

Melia, F., \& Fatuzzo, M. 1992, ApJ, 398, L85

Mészáros, P. \& Rees, M. J. 1992, ApJ, 405, 278

Narayan, R., Paczyński, B., \& Piran, T. 1992, ApJ, 395, L83

Nemiroff, R. J. 1994, Comm. on Astrophys., in press

Nemiroff, R. J., Norris, J. P., Kouveliotou, C., Fishman, G. J., Meegan, C. A., \& Paciesas, W. S. 1994, ApJ, 423, 432

Norris, J. P., Share, G. H., Messina, D. C., Dennis, B. R., Desai, U. I., Cline, T. L., Matz, S. M., \& Chupp, E. L. 1986, ApJ, 301, 213

Norris, J. P., Nemiroff, R. J., Davis, S. P., Kouveliotou, C., Fishman, G. J., Meegan, C. A., \& Paciesas, W. S. 1994, in Proc. of the Huntsville Gamma-Ray Burst Workshop, eds. G. J. Fishman, K. C. Hurley, \& J. J. Brainerd (New York: AIP), in press

Paczyński, B. 1986, ApJ, 308, L43

Paczyński, B. 1988, ApJ, 335, 525

Paczyński, B. 1990, ApJ, 363, 218 
Pendleton, G. N., Paciesas, W. S., Lestrade, J. P., Fishman, G. J., Wilson, R. B., \& Meegan, C. A. 1989, in Proc. Gamma Ray Observatory Science Workshop (Washington: NASA), 4-547

Pendleton, G. N., et al. 1992, The Compton Observatory Science Workshop (Washington:NASA), 47

Press, W. H., Flannery, B. P., Teukolsky, S. A., Vetterling, W. T. 1989, Numerical Recipes (New York: Cambridge)

Schaefer, B. E., 1994, in Proc. of the Huntsville Gamma-Ray Burst Workshop, eds. G. J. Fishman, K. C. Hurley, \& J. J. Brainerd (New York: AIP), in press

Schneid, E. J., et al. 1992, A\&A, 255, L13

Smith, I. A., \& Lamb, D. Q. 1993, in Compton Gamma-Ray Observatory, eds. M. Friedlander, N. Gehrels, \& D. J. Macomb (New York: AIP), p. 1020

Usov, V. V. 1992, Nature, 357, 472

Wheaton, W. A., Dunklee, A. L., Jacobson, A. S., Ling, J. C., Mahoney, W. A., \& Radocinski, R. G. 1994, ApJ, submitted

Woosley, S. E. 1992, ApJ, 405, 273.

This preprint was prepared with the AAS IATEX macros v3.0.

Fig. 1. $-E_{P}$ as determined from spectra at the finest available time resolution (crosses) and from pairs of spectra averaged together (diamonds). The data are from burst 2B910807 and the size of the symbols represents the time intervals over which spectra were accumulated and the error in $E_{P}$. 
Fig. 2.- $E_{P}$ as determined from fits to the same spectra with different binnings for burst 3B930916. The crosses represent values from the unrebinned data while the diamonds correspond to the values from spectra which have been rebinned into eight broad energy bins.

Fig. 3.- $E_{P}$ as measured by detectors at different gains for burst 2B910503. The high gain detector (crosses) was fit from 25.7-2977 keV and the low gain detector (diamonds) covers $308-26,214 \mathrm{keV}$. The high gain detector has better time resolution since it had a stronger signal.

Fig. 4.- $E_{P}$ as determined from two detectors viewing the burst 2B921207. The high gain detector (crosses) covered the energy range from 20.2-1283 keV and the low gain detector (diamonds) ranged from 43.1-3300 keV.

Fig. 5.- The evolution of $E_{P}$ and the count rate for all bursts in the sample. The histogram is the count rate for individual SHERB spectra and the diamonds are $E_{P}$. The height of the diamonds represent 1- $\sigma$ error bars and their width indicates the accumulation time of the SHERB spectra averaged together to achieve the $\mathrm{S} / \mathrm{N}$ criterion outlined in $\S 3$. Note: 3B931204 extends over two frames.

Fig. 6. - A comparison of the peak in $E_{P}$ between intensity pulses within the same burst. The diagonal line indicates equality. Harder early pulses lie below this line and harder late pulses above it. The bursts used are those for which a,+- , or 0 answer could be determined for column 4 in Table 2. Several points line up vertically and horizontally since early pulses were individually compared to all those which followed.

Fig. 7.- The range in $E_{P}$ for a given emission time for all bursts in the sample. The vertical bars give the range in $E_{P}$ (max-min) and the horizontal bars show the period during which significant emission occurred relative to trigger. The crosses mark median values. 